\section{Primary stenting in acute carotid dissection}

\section{Sir,}

A 49-year-old man presented to us with aphasia and right hemiplegia of $6 \mathrm{~h}$ duration. On examination he had a gaze preference to the left side, global aphasia and dense right hemiplegia with Grade 0/5 power. He had no history of trauma or risk factors for stroke. Computerized tomography (CT) scan of brain showed a hyperdensity in the left sylvian fissure suggestive of thrombosis in middle cerebral artery (MCA) branches [Figure 1]. He was taken up for digital substraction angiography (DSA) which showed a tapering occlusion of the left internal carotid artery (ICA) $3 \mathrm{~cm}$ after the origin with absent intracranial flow and very poor collateral flow via the contralateral ICA at $6 \mathrm{~h}$. In view of the mechanical occlusion and the elapsing of the time window for intra-arterial thrombolysis, primary stenting was offered as an option. A microcatheter and microwire were passed through the true lumen of the ICA and a

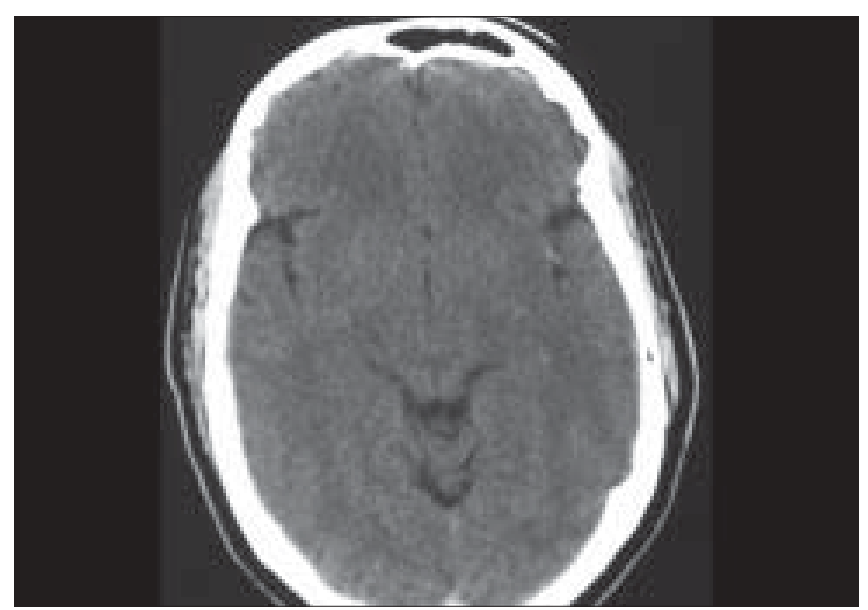

Figure 1: Initial CT showing hyperdense MCA branches in the left sylvian fissure single tapering self-expanding nitinol $8>6 \times 30 \mathrm{~mm}$ Protégé stent was deployed across the lesion by $7 \mathrm{~h}$ after the onset of stroke. Post-deployment angiogram showed excellent recanalisation of the ICA with filling of the left anterior cerebral artery (ACA) and superior division of the MCA [Figure 2]. Further thrombolysis of the inferior division was not attempted. Diffusion weighted magnetic resonance imaging (DWMRI) on Day 3 showed only a periventricular infarction in the left MCA territory [Figure 3]. CT angiography on Day 3 demonstrated the stent in situ with recanalisation of

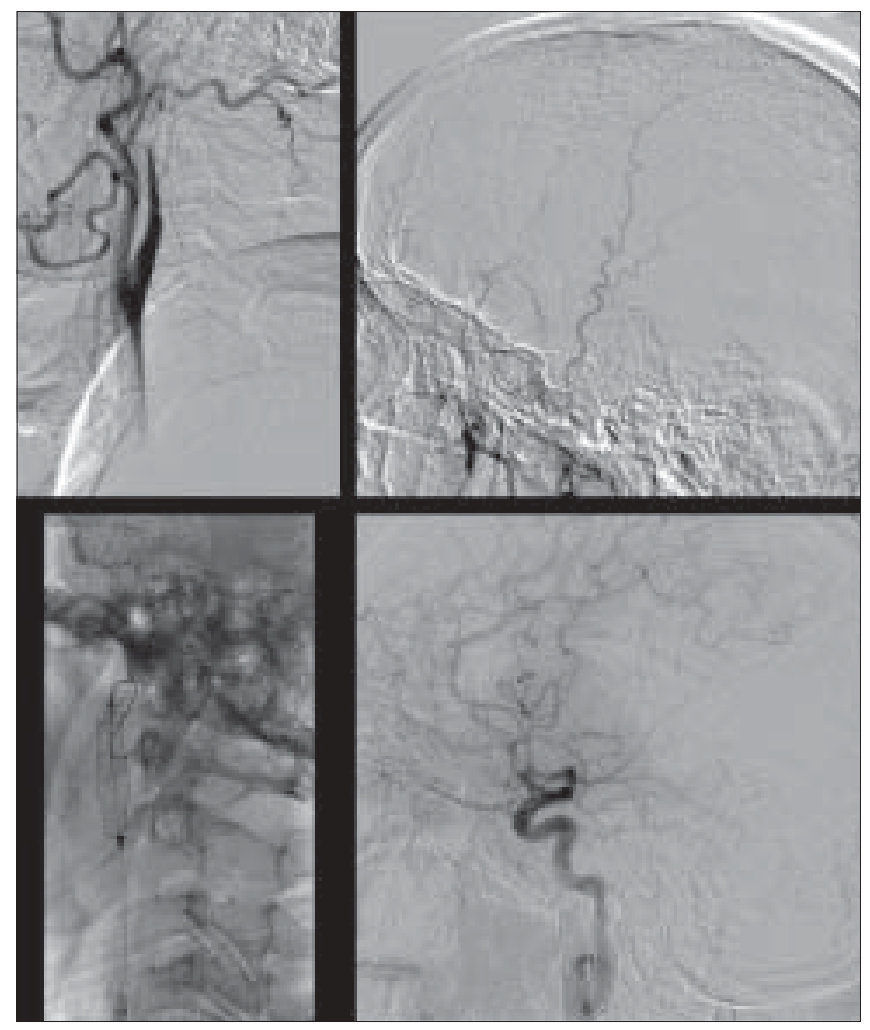

Figure 2: Top panel first image (from left to right) shows dissection of the LICA. Second image shows the absent intracranial flow. Bottom panel first image shows the deployed stent in the LICA. Second image shows the recanalisation of ACA and superior MCA division

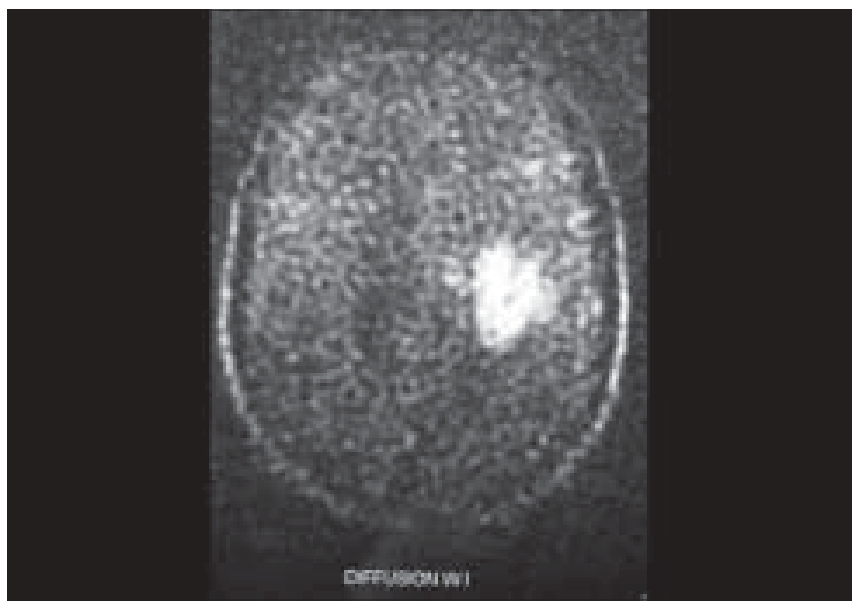

Figure 3: Post-stenting DWMRI showing a periventricular infarct 


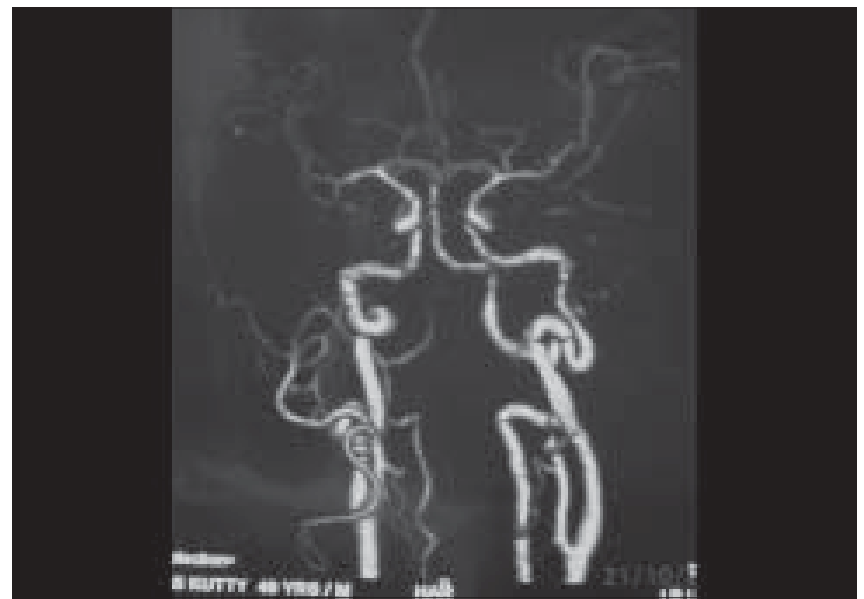

Figure 4: CT angiogram shows the stent in situ (arrow) with recanalisation of the distal ICA

the left ICA [Figure 4]. By Day 3, his lower limb power had improved to Grade 4 and he had only mild residual motor aphasia. At one month, he had Grade 5 power in his right leg and Grade 4 power in his right arm.

Spontaneous internal carotid artery dissection (SICAD) has classically been associated with a good prognosis and many clinicians treat it conservatively. ${ }^{[1]}$ However, a recent study reiterates the very poor recanalization rates in SICAD with complete occlusion. ${ }^{[2]}$ Moreover, collateral flow is critically important in maintaining cerebral perfusion in such patients. Hence our case highlights the option of primary stenting in selected cases of SICAD with ischemic stroke, especially those with complete occlusion of the ICA and poor collateralisation. Prior case reports have shown the importance of considering primary stenting over intraarterial thrombolysis in selected cases of ischemic stroke..$^{[3,4]}$ Intravenous or intra-arterial thrombolysis was unlikely to have provided any benefits in this instance.

Boby Varkey M aramattom Department of Neurology and Interventional Neurology, Lourdes Heart Institute and Neuro Center, Kochi, Kerala, South India, India. E-mail: bobvarkey@gmail.com

DOI: $10.4103 / 0028-3886.48800$

\section{References}

1. Georgiadis D, Caso V, Baumgartner RW. Acute therapy and prevention of stroke in spontaneous carotid dissection. Clin Exp Hypertens $2006 ; 28: 365-70$.

2. Nedeltchev K, Bickel S, Arnold M, Sarikaya H, Georgiadis D, Sturzenegger M, et al. R2-Recanalization of spontaneous carotid artery dissection. Stroke 2008 Dec 24 (epub ahead of print).

3. Janjua N, Qureshi AI, Kirmani J, Pullicino P. Stent-supported angioplasty for acute stroke caused by carotid dissection. Neurocrit Care 2006;4:47-53.

4. Malek AM, Higashida RT, Phatouros CC, Lempert TE, Meyers PM, Smith WS, et al. Endovascular management of extracranial carotid artery dissection achieved using stent angioplasty. AJNR Am J Neuroradiol 2000;21:1280-92.

Accepted on 26-01-2009 